\title{
Intimal Sarcoma of the Pulmonary Artery Treated with Pazopanib
}

\author{
Yohei Funatsu ${ }^{1}$, Miwa Hirayama ${ }^{1}$, Junichi Shiraishi ${ }^{2}$, Takanori Asakura ${ }^{1}$, Misa Wakaki ${ }^{1}$, \\ Erina Yamada ${ }^{1}$, Kazuyuki Fujimoto ${ }^{1}$, Ryosuke Satomi ${ }^{1}$, Shunsuke Inaki ${ }^{1}$, \\ Yuya Murata $^{2}$ and Yoshitaka Oyamada ${ }^{1}$
}

\begin{abstract}
Intimal sarcoma is a rare disease with a poor prognosis. We herein report the case of a 71-year-old man with intimal sarcoma of the pulmonary artery treated with pazopanib. The tumor showed regression after 1 month of treatment. Hand-foot syndrome led to cessation of pazopanib, which triggered a disease flare. Pazopanib should be considered in patients with intimal sarcoma of the pulmonary artery that is unresectable or recurrent after surgery or cytotoxic chemotherapy. We must be careful about drug cessation, as it can lead to a disease flare.
\end{abstract}

Key words: intimal sarcoma of the pulmonary artery, pazopanib, disease flare, tyrosine kinase inhibitor

(Intern Med 55: 2197-2202, 2016)

(DOI: 10.2169/internalmedicine.55.6199)

\section{Introduction}

Intimal sarcoma is a rare disease with a poor prognosis. The median survival has been reported to be $36.5 \pm 20.2$ months for patients who underwent curative resection, compared with $11 \pm 3$ months for those who underwent incomplete resection (1). Due to its clinical manifestation, which resembles pulmonary embolism, intimal sarcoma is often misdiagnosed. We herein report the case of a 71-year-old man with intimal sarcoma of the pulmonary artery treated with pazopanib, a multitargeted tyrosine kinase inhibitor (TKI), showing a partial response to treatment. Hand-foot syndrome led to cessation of pazopanib, however. After cessation of pazopanib, the patient demonstrated a rebound effect, or a disease flare, similar to that observed in a lung cancer patient who was treated with an epidermal growth factor receptor TKI (2-4).

\section{Case Report}

A 71-year-old man was referred to our hospital presenting with dyspnea on exertion. His medical history included hypertension, lower extremity varix, appendicitis, tuberculous lymphadenitis, and a 30 pack-year history of smoking. Plain chest computed tomography (CT) showed old inflammatory scarring of the lung, while contrast-enhanced CT revealed saddle embolism and thrombus in both the left and right main pulmonary arteries (Fig. 1). The patient's body temperature, heart rate, and blood pressure on admission were $36.8^{\circ} \mathrm{C}, 78$ beats/minute, and 129/60 $\mathrm{mmHg}$, respectively. Echocardiography showed a normal right ventricle chamber size, right atrial systolic pressure of $10 \mathrm{mmHg}$, tricuspid regurgitation pressure gradient of $46.1 \mathrm{mmHg}$, right ventricular systolic pressure of $46.1 \mathrm{mmHg}$, and inferior vena cava diameter of $19 \mathrm{~mm}$, with respiratory variation.

These findings suggested pulmonary embolism, and anticoagulation therapy was initiated. The defect remained despite treatment, however, and his dyspnea persisted. Therefore, surgical resection of the intraluminal lesion was performed. The resected tissue was an intravascular polypoid mass extending distally along the branches of the vessels and appeared to be a soft tumor-like mass rather than a thrombus. Histologic findings revealed spindle cell sarcoma

${ }^{1}$ Respiratory Medicine, National Hospital Organization Tokyo Medical Center, Japan and ${ }^{2}$ Pathology, National Hospital Organization Tokyo Medical Center, Japan

Received for publication July 16, 2015; Accepted for publication November 29, 2015

Correspondence to Dr. Yohei Funatsu, dodeko81@ybb.ne.jp 

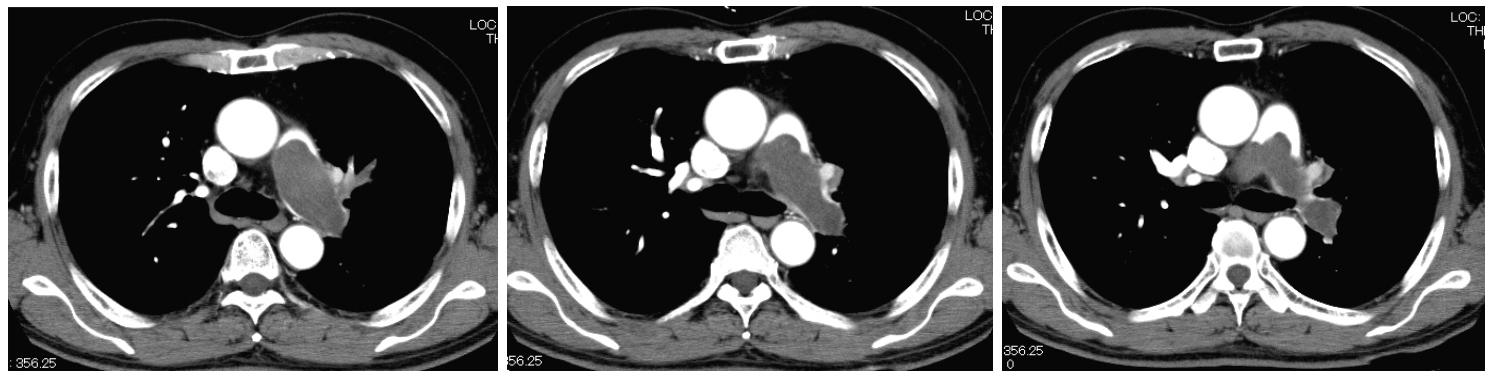

Figure 1. Contrast-enhanced computed tomography scans showing saddle embolism and thrombus in both the left and right main pulmonary arteries.
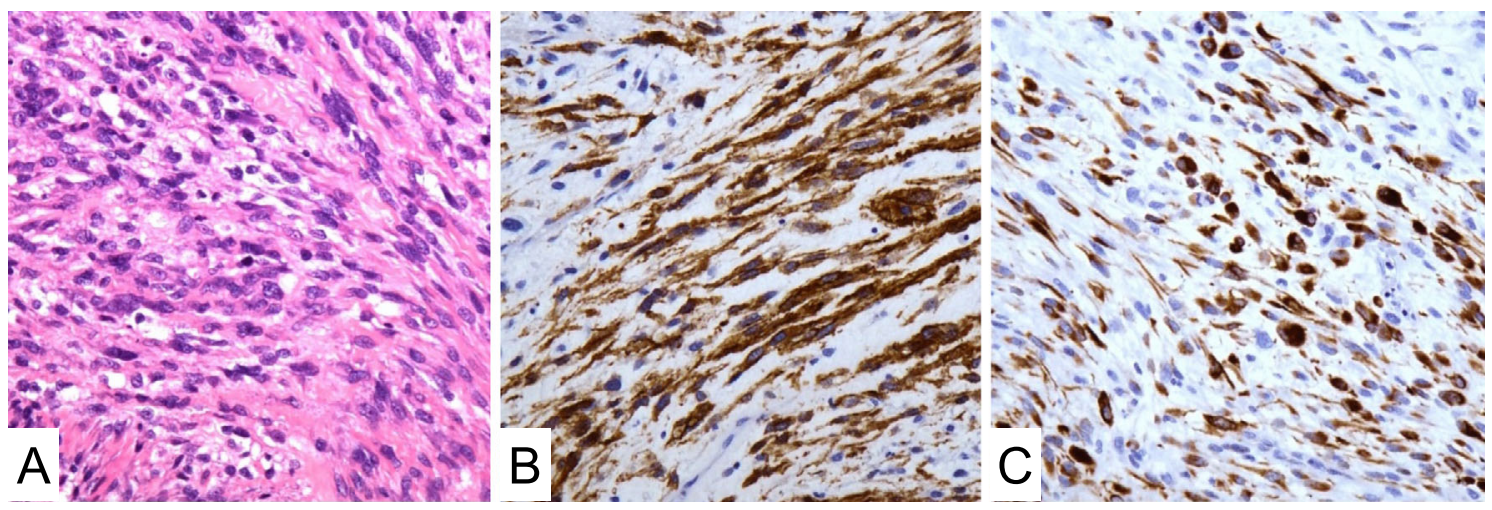

Figure 2. (A) A histopathologic examination revealing atypical spindle cells with high cellularity and nuclear pleomorphism. (B) Immunohistochemical staining showing focally positive results for alpha-smooth muscle actin. (C) Immunohistochemical staining showing focally positive results for desmin.

with necrosis, mitotic activity, and nuclear pleomorphism. Immunohistochemical staining was focally positive for smooth muscle actin and desmin, but negative for CD31, CD34, S-100, and cytokeratin (CK) AE1/AE3. According to these findings, the tumor was diagnosed as intimal sarcoma (Fig. 2). The tumor was completely resected. Positron emission tomography-CT performed 1 month later showed no aggregation of fluorodeoxyglucose in the pulmonary artery.

Follow-up CT performed 3 months after resection revealed an intraluminal main pulmonary arterial mass invading the left pulmonary artery trunk, as well as a mass in the left lung. According to these findings, local recurrence of intimal sarcoma of the pulmonary artery was diagnosed, with suspected metastasis to the left lung.

The patient was admitted to our hospital to receive chemotherapy. On the first day of admission, he complained of acute chest pain. Contrast-enhanced CT showed left pleural effusion and rapid enlargement of the tumor in the left pulmonary artery trunk, indicating bleeding into the tumor and the left pleural space. An analgesic was administered as palliative therapy and the symptom gradually subsided.

Combination chemotherapy consisting of ifosfamide $(2.5$ $\mathrm{g} / \mathrm{m}^{2}$ on days $\left.1-4\right)$ and doxorubicin (25 $\mathrm{mg} / \mathrm{m}^{2}$ on days $1-3$ ) every 3 weeks was initiated. However, the patient suffered serious hematologic (grade 3 neutropenia) and nonhematologic (grade 3 fatigue) toxicity, and he refused to continue treatment. One month after starting first-line chemotherapy, CT revealed enlargement of the tumor, indicating progressive disease. Therefore, pazopanib $800 \mathrm{mg} /$ day was started as second-line chemotherapy. One month later, shrinkage of the tumor (Fig. 3), which met the criteria of a partial response, was demonstrated on CT. The following month, progressively worsening blisters appeared on both palms, and he was diagnosed as having hand-foot syndrome caused by pazopanib. Two months after starting pazopanib, the tumor again showed enlargement. However, we continued pazopanib therapy due to the lack of alternatives and because tumor growth suppression was still anticipated (Fig. 4).

Four months after starting pazopanib, the patient required cessation of pazopanib because aggravation of his hand-foot syndrome made it impossible to walk (Fig. 5). One week after cessation, the patient was admitted to the hospital for an emergency due to hemothorax (Fig. 6) and increasing pain; a disease flare was subsequently suspected. Meanwhile, discontinuation of the drug for 1 week improved his hand-foot syndrome, so pazopanib was started again. Three days after readministration of the agent, no further increase in pleural effusion or tumor size was recognized. Twelve months after the diagnosis, the patient died of intimal sarcoma.

\section{Autopsy findings}

The tumor relapsed at the site of resection, invaded into 

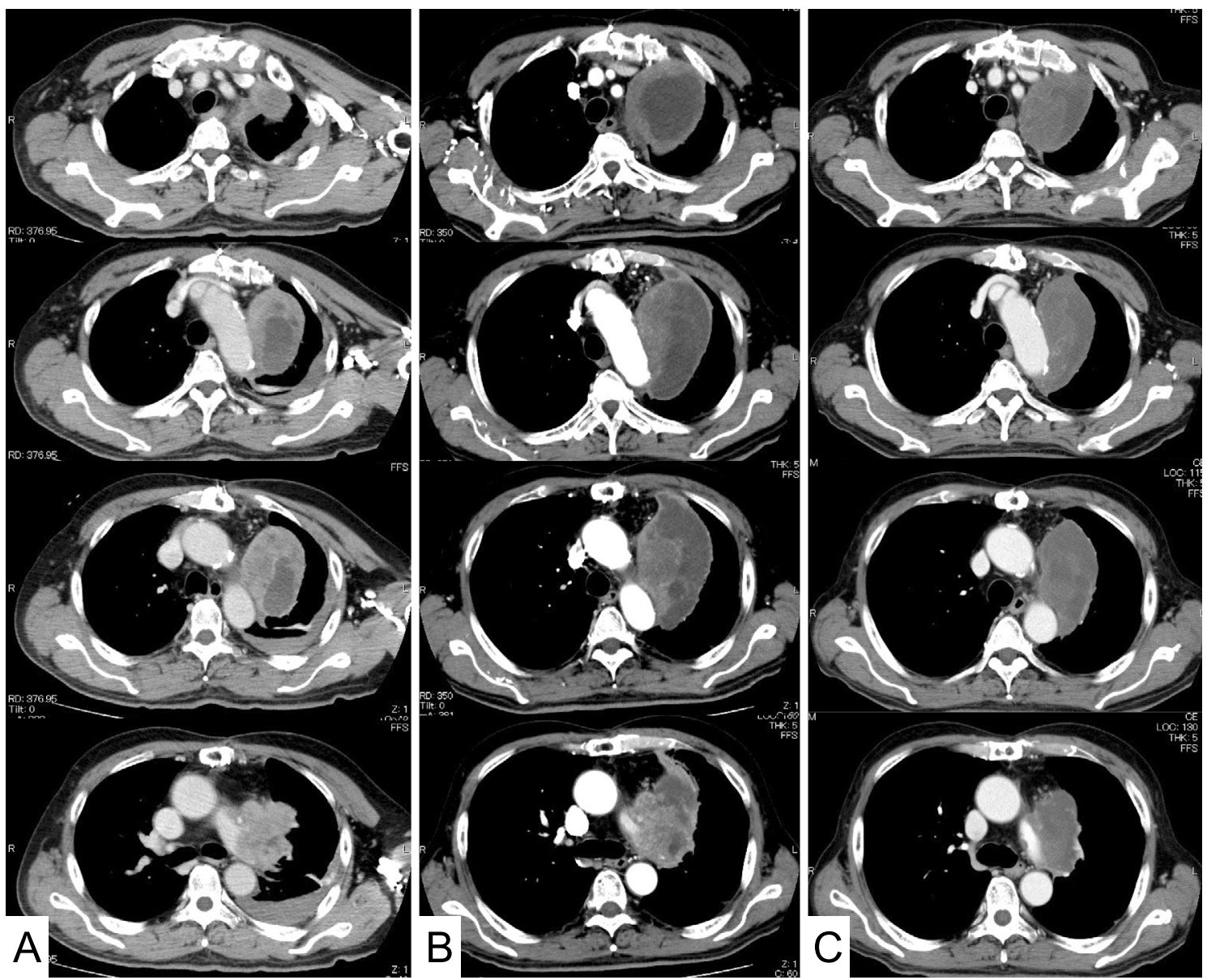

Figure 3. (A) A computed tomography (CT) scan performed 7 days before first-line chemotherapy, showing invasion to the extrapulmonary artery. (B) A CT scan performed 1 month after first-line chemotherapy. (C) A CT scan performed 1 month after starting pazopanib as second-line chemotherapy.

the left upper lobe of the lung, and extended to the mediastinum and left thoracic cavity. In the left upper lobe of the lung, the tumor progressed along the pulmonary artery and extended to the lung parenchyma with a large cavity. Tumor cells and lung tissue with degeneration and necrosis in the cavity were observed, which were induced not only by ischemic change with tumor enlargement, but also by the effect of chemotherapy (Fig. 7).

Histologically, the tumor consisted of diffuse proliferation of spindle and pleomorphic cells with a vague fascicular storiform pattern and nuclear atypia. Immunohistochemically, the tumor cells were focally positive for smooth muscle actin, vimentin, and Ki-67 but negative for CK (AE1/AE3), CD31, CD34, desmin, myoglobin, S-100, and c-KitR.

\section{Discussion}

Sarcoma of the pulmonary artery is rare and has a poor prognosis, with a median survival of 1.5 months without surgical resection and 10 months with resection (5). Pulmonary artery sarcomas are derived from the intima. The World Health Organization has classified intimal sarcoma as myofibroblastic sarcoma without angiosarcoma.
Although some reports have shown that chemotherapy or radiotherapy is effective against pulmonary artery sarcoma, its role in the treatment of unresectable intimal sarcoma or relapsed sarcoma remains uncertain (6-8). In this case, we used combination doxorubicin and ifosfamide as first-line chemotherapy and pazopanib as second-line chemotherapy. Pazopanib is a multitargeted TKI with a high affinity against vascular endothelial growth factor receptor-1/2/3 and a lower affinity against platelet-derived growth factor receptor- $\alpha / \beta$, fibroblast growth factor receptor-1/2, and stem cell factor receptor c-KitR (9-11). A phase III trial of pazopanib against soft tissue sarcoma was performed in 2012. The effect of pazopanib on patients with metastatic nonadipocytic soft tissue sarcoma was assessed. The median progression-free survival was 4.6 months (range, 3.7-4.8) with pazopanib compared with 1.6 months (range, 0.9-1.8) with placebo [hazard ratio (HR), 0.31 ; $95 \%$ confidence interval (CI), 0.24-0.40; $\mathrm{p}$ $<0.0001]$. The overall survival was 12.5 months (range, 10.6-14.8) with pazopanib versus 10.7 months (range, 8.712.8) with placebo (HR, 0.86; 95\% CI, 0.67-1.11; p= $0.25)$ (12). In 2012, pazopanib was approved by the Ministry of Health, Labour and Welfare of Japan.

In this case, initial tumor shrinkage met the criteria for a 

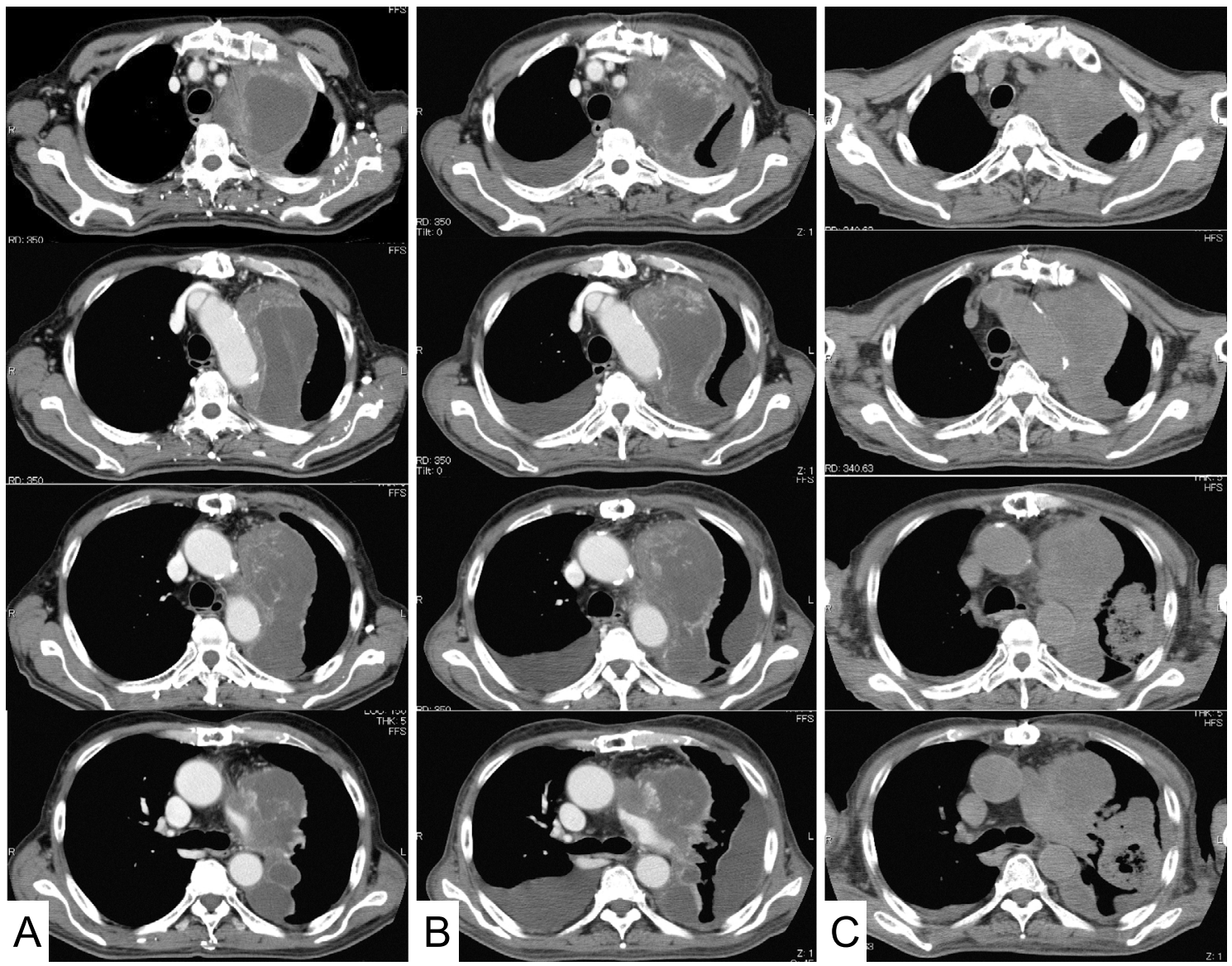

Figure 4. (A) A Computed tomography (CT) scan performed 2 months after starting pazopanib. (B) A CT scan performed 1 month after disease flare, 4 months after starting pazopanib. (C) A CT scan performed 2 months after disease flare, 5 months after starting pazopanib.

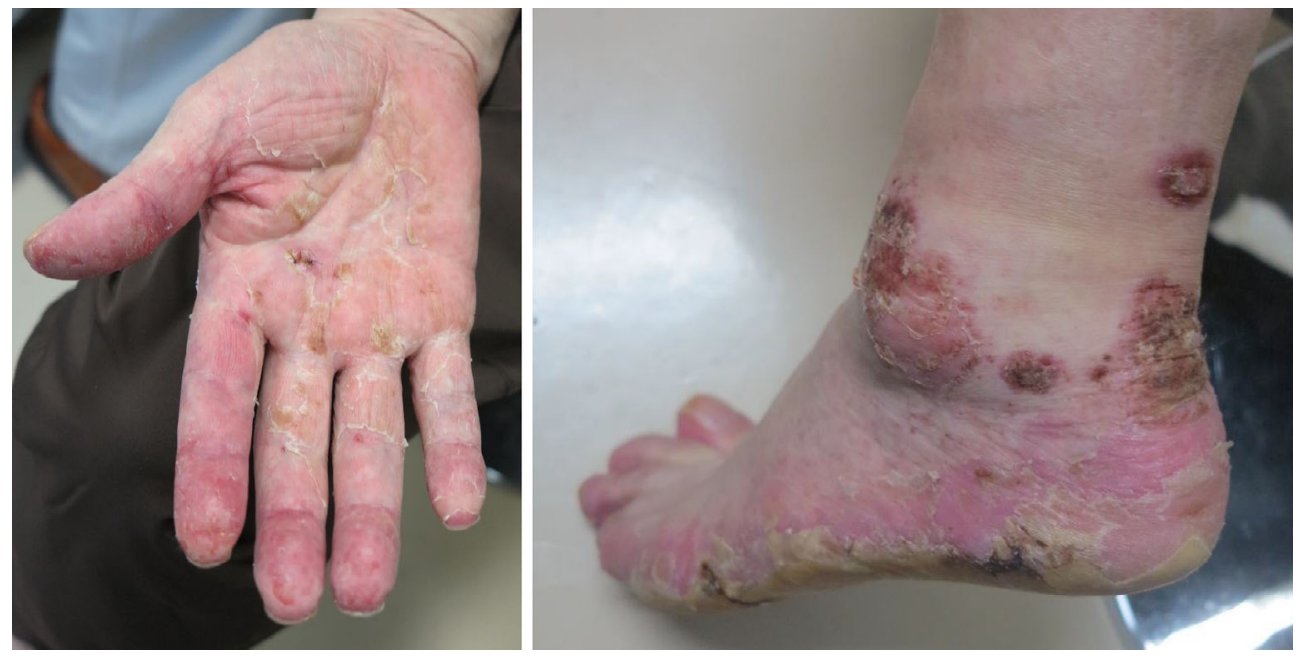

Figure 5. Photographs showing hand-foot syndrome as an adverse effect of pazopanib.

partial response. Thus, pazopanib was thought to be at least transiently effective. Rapid enlargement of the tumor was observed during the period of pazopanib cessation, indicating a rebound flare. Although a disease flare due to cessation of epithelial growth factor receptor-TKI for lung cancer has been reported, a disease flare due to cessation of TKI for intimal sarcoma has not.
The efficacy of chemotherapy for the treatment of intimal sarcoma is limited; thus, pazopanib should be considered in patients with sarcoma of the pulmonary artery that is unresectable or recurrent after surgery or cytotoxic chemotherapy. In such cases, we should be careful about drug cessation because it can lead to a disease flare. However, in cases where the interruption of therapy is unavoidable, we should 


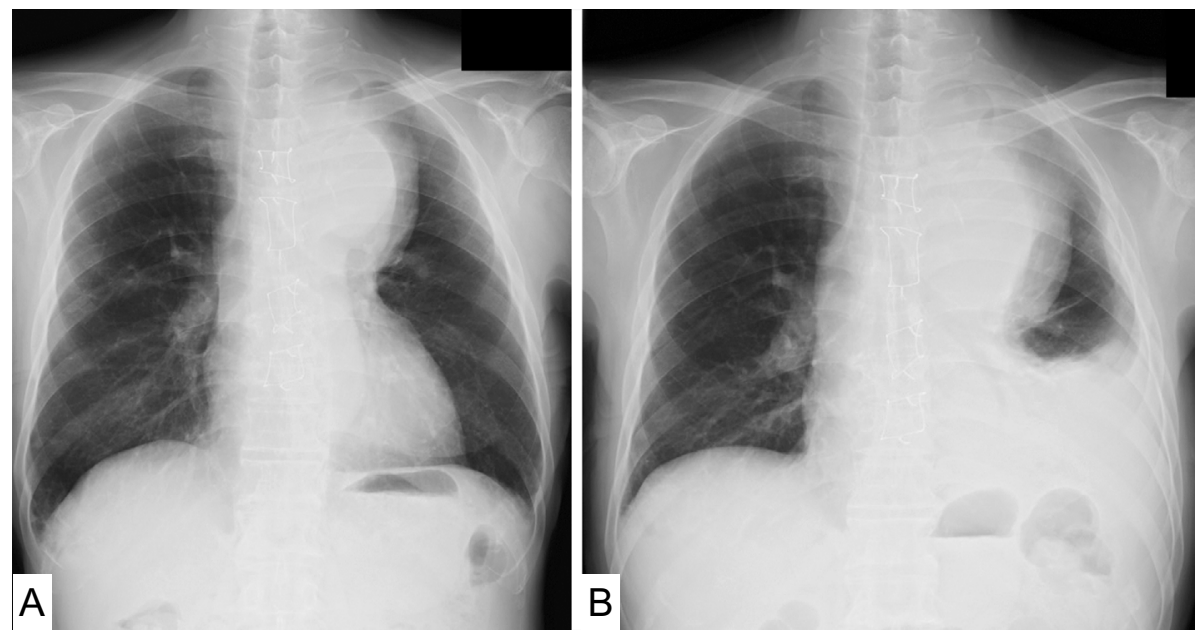

Figure 6. (A) A chest radiograph performed 1 and a half months after starting pazopanib. (B) A chest radiograph performed 1 week after cessation of pazopanib, showing increased pleural fluid.

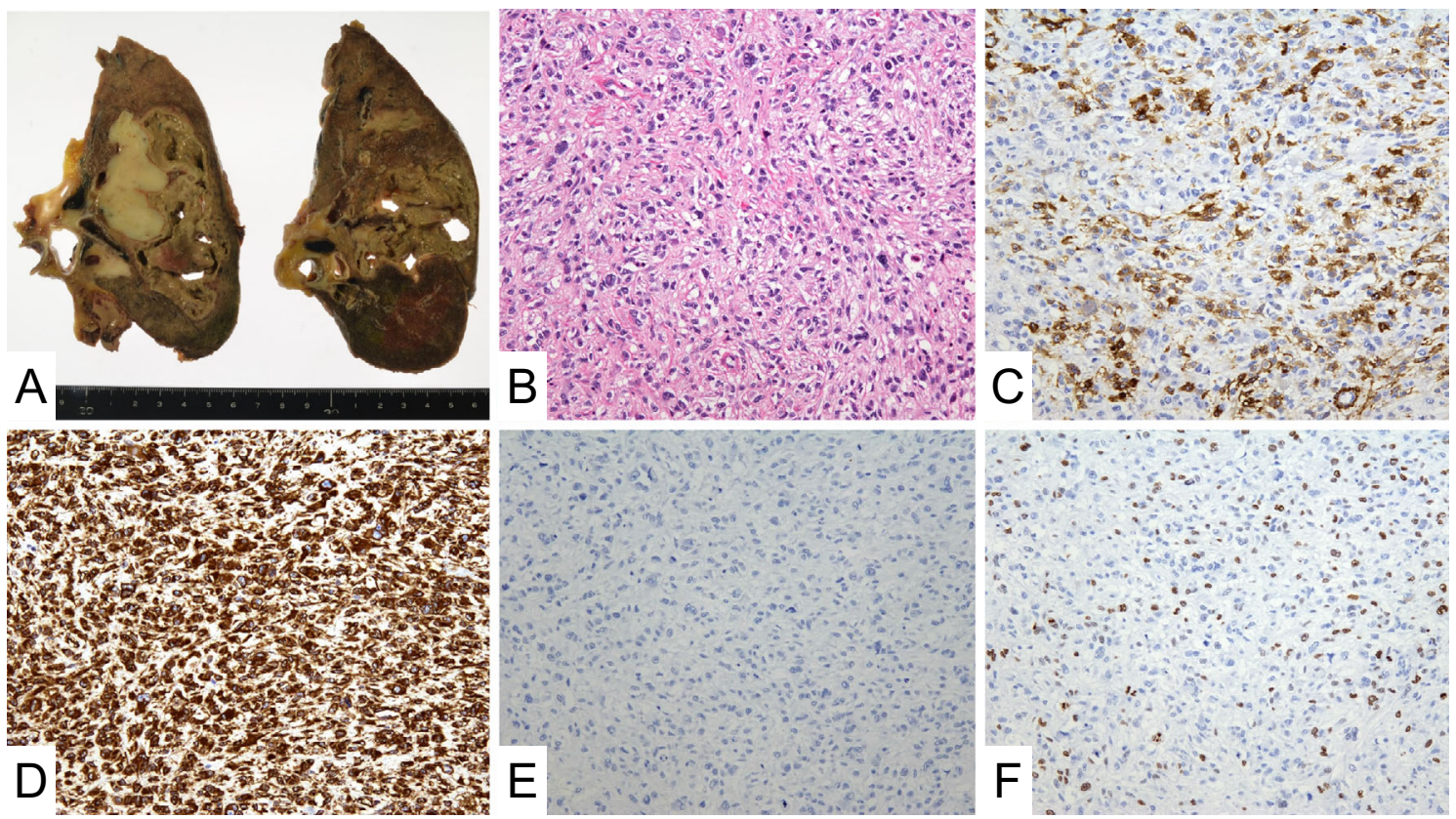

Figure 7. (A) Gross pathologic appearance of the tumor protruding into the left pulmonary artery. (B) A histopathologic examination revealing atypical spindle cells with high cellularity and nuclear pleomorphism. (C) Immunohistochemical staining showing focally positive results for smooth muscle actin. (D) Immunohistochemical staining showing diffuse positive results for vimentin. (E) Immunohistochemical staining showing negative results for desmin. (F) Immunohistochemical staining showing focally positive results for Ki-67.

make an effort to minimize the period of interruption. The use of the drug beyond progressive disease is also a potential option.

The authors state that they have no Conflict of Interest (COI).

\section{References}

1. Blackmon SH, Rice DC, Correa AM, et al. Management of primary pulmonary artery sarcomas. Ann Thorac Surg 87: 977-984, 2009.
2. Pop O, Pirvu A, Toffart AC, Moro-Sibilot D. Disease flare after treatment discontinuation in a patient with EML4-ALK lung cancer and acquired resistance to crizotinib. J Thorac Oncol 7: e1-e2, 2012.

3. Chaft JE, Oxnard GR, Sima CS, Kris MG, Miller VA, Riely GJ. Disease flare after tyrosine kinase inhibitor discontinuation in patients with EGFR-mutant lung cancer and acquired resistance to erlotinib or gefitinib: implications for clinical trial design. Clin Cancer Res 17: 6298-6303, 2011.

4. Kim YH, Fukuhara A, Mishima M. Should epidermal growth factor receptor-tyrosine kinase inhibitor be continued beyond progressive disease? Case Rep Oncol 4: 470-474, 2011. 
5. Chen X, Ren S, Li A, Zhou C. A case report of chemo-sensitive intimal pulmonary artery sarcoma. Cell Biochem Biophys 68: 153157, 2014.

6. Uchida A, Tabata M, Kiura K, et al. Successful treatment of pulmonary artery sarcoma by a two-drug combination chemotherapy consisting of ifosfamide and epirubicin. Jpn J Clin Oncol 35: 417419, 2005.

7. Head HD, Flam MS, John MJ, Lipnik SS, Slater DL, Stewart RD. Long-term palliation of pulmonary artery sarcoma by radical excision and adjuvant therapy. Ann Thorac Surg 53: 332-334, 1992.

8. Majeski J, Crawford ES, Majeski EI, Duttenhaver JR. Primary aortic intimal sarcoma of the endothelial cell type with long-term survival. J Vasc Surg 27: 555-558, 1998.

9. Ranieri G, Pantaleo M, Piccinno M, et al. Tyrosine kinase inhibi- tors (TKIs) in human and pet tumours with special reference to breast cancer: a comparative review. Crit Rev Oncol Hematol 88: 293-308, 2013.

10. Ranieri G, Gadaleta CD, Patruno R, et al. A model of study for human cancer: spontaneous occurring tumors in dogs. Biological features and translation for new anticancer therapies. Crit Rev Oncol Hematol 88: 187-197, 2013.

11. Folkman J. Anti-angiogenesis: new concept for therapy of solid tumors. Ann Surg 175: 409-416, 1972.

12. van der Graaf WT, Blay JY, Chawla SP, et al; EORTC Soft Tissue and Bone Sarcoma Group; PALETTE study group. Pazopanib for metastatic soft-tissue sarcoma (PALETTE): a randomised, doubleblind, placebo-controlled phase 3 trial. Lancet 379: 1879-1886, 2012.

(C) 2016 The Japanese Society of Internal Medicine http://www.naika.or.jp/imonline/index.html 